\title{
New NCCN Guidelines: Smoking Cessation for Patients With Cancer
}

Presented by Peter G. Shields, MD

\begin{abstract}
Even after a cancer diagnosis, for any stage and prognosis, patients with cancer can reap health benefits from smoking cessation. To address the clinical problems associated with smoking in cancer patients, the NCCN created a new set of NCCN Clinical Practice Guidelines in Oncology (NCCN Guidelines) for 2015. At NCCN 20th Annual Conference, Dr. Peter Shields presented these inaugural guidelines, focusing primarily on the adverse outcomes associated with smoking in patients with cancer, the common barriers to smoking cessation in this patient population, simple yet effective assessment approaches, and treatment recommendations that center on the combination of pharmacotherapy and behavioral therapy. (J Natl Compr Canc Netw 2015;13:643-645)
\end{abstract}

Only $50 \%$ of oncologists advise their patients to quit smoking, and most of them do not provide steps on how to do it, stated Peter G. Shields, MD, Professor in the Division of Medical Oncology, Department of Medicine in the College of Medicine and in the Division of Epidemiology in the College of Public Health at The Ohio State University; Deputy Director of The Ohio State University Comprehensive Cancer Center - James Cancer Hospital and Solove Research Institute; and Chair of the NCCN GuidelinesPanel on Smoking Cessation.

"Treating patients with cancer is not simple, and smoking issues easily take second or third or fourth priority, so

Peter G. Shields, MD, is a Professor in the Department of Internal Medicine, Division of Medical Oncology at The Ohio State University Comprehensive Cancer Center - James Cancer Hospital and Solove Research Institute and Wexner Medical Center at The Ohio State University, Columbus, Ohio.

Dr. Shields has disclosed that he has no financial interests, arrangement, affiliations, or commercial interests with the manufacturers of any products discussed in this article or their competitors.

Correspondence: Peter G. Shields, MD, The Ohio State University Comprehensive Cancer Center - James Cancer Hospital and Solove Research Institute; Wexner Medical Center at The Ohio State University, 460 W 10th Avenue, 9th Floor, Suite D920, Columbus, $\mathrm{OH}$ 43210-1240.

E-mail: peter.shields@osumc.edu we wanted to come up with guidelines that would be clear, relevant, and something that could literally be done in the clinic in just a few minutes," Dr. Shields said. The goals of the new NCCN Clinical Practice Guidelines in Oncology (NCCN Guidelines) for Smoking Cessation are to establish standards of care, facilitate implementation with clear guidelines, allow for quality-control monitoring, fill a gap among current guidelines, and ultimately improve the health and outcomes of patients with cancer.

\section{Rationale Behind Creation of Smoking Guidelines}

Smoking is a real problem in patients with cancer, and it can and should be addressed effectively, Dr. Shields noted. The American Association for Cancer Research Taskforce on Tobacco noted that almost no institutions have reported systematic and consistent mechanisms for fostering smoking cessation, explained Dr. Shields, and we need to do a better job in the clinic to help patients with cancer kick the habit.

According to the 2014 Surgeon General Report on Smoking and Health, ${ }^{1}$ sufficient evidence exists of a causal relationship between adverse health outcomes and smoking, including an increase in all-cause mortality, cancerspecific mortality, and risk for second primary cancers. In addition, it noted, smoking cessation improves prognosis and reduces toxicities for many of our treatments.

Dr. Shields briefly discussed some of the long-standing and recent data behind the negative effects of smoking on patients with cancer. In 1993, Browman et $\mathrm{al}^{2}$ evaluated the influence of cigarette smoking on the efficacy of radiation therapy in head and neck cancer. "There were clear differences in survival between long-time quitters of smoking and those who continued to smoke," revealed Dr. Shields.

In 2009, Zevallos et $\mathrm{al}^{3}$ focused on the effects of a prospective smoking cessation program on complications of 
radiotherapy in patients with laryngopharyngeal cancer. The investigators found that smokers experienced more hospitalizations during treatment, more pharyngeal strictures requiring dilatation, and more osteoradionecrosis needing major resection than did nonsmokers.

And more recently, in 2014, O'Malley et $\mathrm{al}^{4}$ studied the effects of cigarette smoking on the metabolism and effectiveness of systemic therapies for lung cancer. Due to the narrow therapeutic index of systemic therapies for lung cancer, the authors concluded that smokinginduced changes in drug plasma concentrations could reduce treatment efficacy and negatively impact outcomes.

\section{Barriers to Smoking Cessation}

Several common barriers to smoking cessation efforts in patients with cancer have been cited by oncologists, including lack of time and priority, interventions that are too seemingly complex, lack of adequate training and expertise, and the mistaken belief that patients with cancer may not benefit enough from brief physician advice, detailed Dr. Shields.

Patients with cancer who smoke indeed represent a unique population of smokers, and there are challenges to speedy smoking cessation. This patient population may have high psychological stress and depression, complicating the clinical picture even further.

However, according to Dr. Shields, "We should take advantage of this teachable moment," and the new NCCN Guidelines for Smoking Cessation offer clear steps for patient assessment, recommend evidence-based pharmacotherapy (Figure 1) and behavioral therapy, stress the importance of close follow-up and adequate documentation in the medical record, and present a variety of resources for patients and providers (quit lines, online support, mobile apps, and training modules; Figure 2).

\section{Highlights of the New Guidelines}

In the new NCCN Guidelines, initial assessment of patient smoking centers around 2 questions: Have you ever smoked cigarettes? Do you currently smoke cigarettes or have smoked in the past 30 days? These brief questions could be asked by any member of the medical team, including a medical assistant or nurse, said Dr. Shields. The intent of these questions was to make it easy for clinicians to bring up the subject in a few short minutes, he added. With a couple more questions, the health care provider can quickly gauge the challenges for quitting and the risk of relapse.

Then the algorithm provides guidance for evaluat-

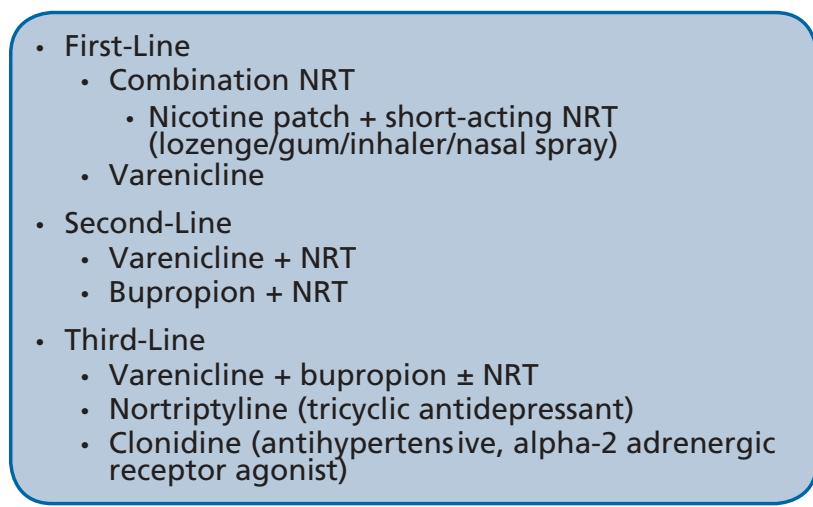

Figure 1 Pharmacotherapy options for smoking cessation. Abbreviation: NRT, nicotine-replacement therapy.

ing 1) current smokers and 2) former smokers or recent quitters (more than 30 days). The evaluation of former smokers focuses on identifying risk factors for smoking relapse, which include the frequency and intensity of cravings, elevated stress and/or depression, living/working with smokers, current use of a smoking cessation treatment, and drug use/abuse.

For current smokers, the first step is to determine their readiness to quit smoking. For instance, the patient should be asked whether he or she is willing to make a quit attempt in the next 30 days.

For those who are ready to quit, providers should assess the challenges and barriers for quitting, and document these in the medical record, including the patient's nicotine dependency by asking the following questions: How many cigarettes do you smoke in a day? How soon do you smoke after you wake up in the morning? Are you living with smokers? The history of quit attempts should also be documented. "The more nicotine dependent they are, the harder it will be for them to quit, and the more aggressive you will have to be with them," explained Dr. Shields. A personalized quit plan should be established, a quit date set, treatment options presented, and risk of relapse discussed.

For those who are not ready to quit, Dr. Shields encouraged oncologists to engage in motivational talk about smoking cessation, reviewing the health risks of smoking and the benefits of quitting and providing patient education. In addition, a patient's readiness to quit should be reassessed at each visit.

For patients who are still smoking and say they want to quit, or for persons who have recently quit smoking, Dr. Shields noted that pharmacotherapy (such as shortacting nicotine replacement therapy [NRT]) and behavior therapy can be considered. This also would be the time to review the risks associated with smoking and benefits of abstinence and to offer patient support resources. Dr. Shields also stressed the importance of fol- 


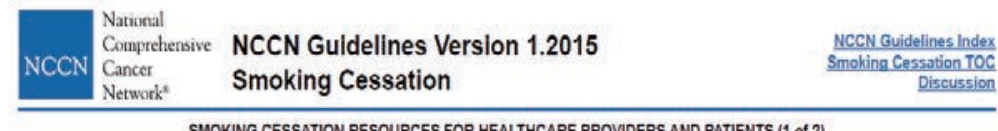

SMOKING CESSATION RESOURCES FOR HEALTHCARE PROVIDERS AND PATIENTS ( 1 of 2 )

\begin{tabular}{|c|c|}
\hline \multicolumn{2}{|l|}{ uittlines/Online SupportMobille Apps } \\
\hline American Lung Association & 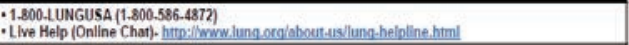 \\
\hline National Network of Tobacco Cessation Quitlines & 1.800-OUIT-HOW (1-800-784-8669) \\
\hline National Cancer Institute (NCI) & 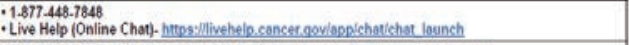 \\
\hline Smokefree.gov & 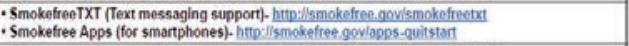 \\
\hline $\begin{array}{l}\text { TRICARE } \\
\text { (For military service members and their families) }\end{array}$ & 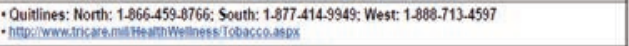 \\
\hline Quit Tobacco: UCANOUITZ org & 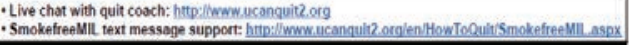 \\
\hline \multicolumn{2}{|l|}{ General Information Online } \\
\hline American Heant Association & 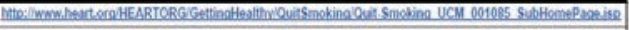 \\
\hline American Lung Assoclation & 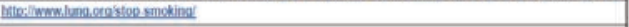 \\
\hline Centers for Disease Control and Prevention (COC) & 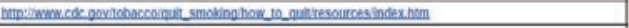 \\
\hline NCI & 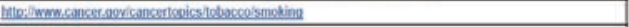 \\
\hline Smokefreegov & 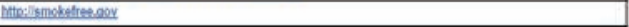 \\
\hline \multicolumn{2}{|l|}{ Smoking Cessation Programs } \\
\hline American Lung Association & 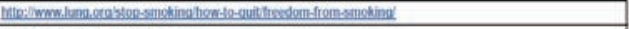 \\
\hline Ex: A Nlew Way To Think About Qultring Smoking & 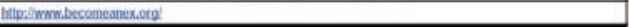 \\
\hline \multicolumn{2}{|l|}{ Guides to Quilting } \\
\hline American Cancer Society (ACS) & 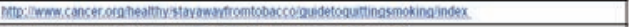 \\
\hline NCL: "Clearing the & \\
\hline
\end{tabular}

Figure 2 Smoking cessation resources.

low-up and reevaluation, either in person or by phone.

Dr. Shields briefly mentioned the growing popularity of electronic cigarettes (e-cigarettes). Currently, the new NCCN Guidelines do not recommend e-cigarettes for smoking cessation because there is insufficient evidence to support their use. "Wall Street and scientists are predicting that these [e-cigarettes] will be the tobacco endgame, replacing cigarettes over the next 10 to 15 years," announced Dr. Shields. Although he noted that their use is less risky than smoking traditional cigarettes, "they are not necessarily safe by themselves," he said, and studies regarding their safety and efficacy are sorely needed. For patients who claim that they have tried everything and nothing has worked, it should be remembered that therapies proven to be effective (eg, combination NRT) may need to be tried several times before a smoker can achieve long-term abstinence.

\section{Therapeutic Options}

According to the new NCCN Guidelines, the recommended first-line therapy is combination NRT (nicotine patch plus nicotine lozenge, gum, inhaler, or nasal spray) or varenicline, which needs to be combined with behavioral therapy (individual or group). In second- or third-line settings, combination NRT can be combined with varenicline, bupropion, or other pharmacotherapies. Dr. Shields stressed that smoking slips and relapses are common and should be expected and worked through. These may not warrant a therapeutic change. Follow-up is critical, he added, within 2 weeks by phone or in person (even e-mail or text). These then reassessment at 12 weeks is suggested.

Dr. Shields emphasized that pharmacotherapy without behavioral therapy is less effective than combination treatment. Actually, pharmacotherapy without any counseling, no matter how brief, is not more effective than no pharmacotherapy. A few minutes of counseling about the benefits and prevention of relapse makes a difference. A recent study by Kotz et $\mathrm{al}^{5}$ looked at the effectiveness of smoking cessation treatments in more than 10,000 smokers who made at least one quit attempt in the prior 12 months. The investigators found that patients who received pharmacotherapy accompanied by behavioral therapy had a more than threefold greater chance of being able to quit compared with patients receiving medication only. Furthermore, for patients who had just NRT over the counter, with no therapy at all, the results were similar to doing nothing at all.

\section{References}

1. U.S. Department of Health and Human Services. The Health Consequences of Smoking-50 Years of Progress: A Report of the Surgeon General, 2014. Available at www.surgeongeneral.gov/library/reports/50-years-of-progress. Accessed March 18, 2015.

2. Browman GP, Wong G, Hodson I, et al. influence of cigarette smoking on the efficacy of radiation therapy in head and neck cancer. N Engl J Med 1993;328:159-163.

3. Zevallos JP, Mallen MJ, Lam CY, et al. Complications of radiotherapy in laryngopharyngeal cancer: effects of a prospective smoking cessation program. Cancer 2009;115:4636-4644.

4. O'Malley M, King AN, Conte M, et al. Effects of cigarette smoking on metabolism and effectiveness of systemic therapy for lung cancer. J Thorac Oncol 2014;9:917-926.

5. Kotz D, Brown J, West R. 'Real-world' effectiveness of smoking cessation treatments: a population study. Addiction 2014;109:491-499. 